\title{
Modelagem matemática da secagem em camada delgada de bagaço de uva fermentado
}

\author{
Luiz Fernando Dias Ferreira(1), Mônica Ribeiro Pirozi ${ }^{(2)}$, Afonso Mota $\operatorname{Ramos}^{(2)}$ e José Antônio Marques Pereira(2) \\ (1)Instituto Federal do Espírito Santo, Campus Venda Nova do Imigrante, Avenida Elizabeth Minete Perim, s/nº, CEP 29375-000 Venda \\ Nova do Imigrante, ES. E-mail: luizfernando@ifes.edu.br (2)Universidade Federal de Viçosa, Departamento de Tecnologia de Alimentos, \\ Avenida P. H. Rolfs, s/no, CEP 36570-000 Viçosa, MG. E-mail: mpirozi@ufv.br, amramos@ufv.br, jamperei@ufv.br
}

\begin{abstract}
Resumo - O objetivo deste trabalho foi determinar as características da secagem de bagaço de uva fermentado em secador com ar aquecido, avaliar a capacidade descritiva de conhecidos modelos matemáticos de secagem em camada delgada, e obter os valores de difusividade efetiva e a energia de ativação. Os experimentos de secagem foram conduzidos a 50, 60, 70, 80 e $90^{\circ} \mathrm{C}$, com a velocidade do ar de secagem de $1,0 \mathrm{~m} \mathrm{~s}^{-1}$. Foram comparados dez diferentes modelos matemáticos de secagem em camada delgada, de acordo com os valores do coeficiente de determinação $\left(\mathrm{R}^{2}\right)$, qui-quadrado $\left(\chi^{2}\right)$, raiz do quadrado médio residual (RQMR) e erro médio relativo (P), estimados pelas curvas de secagem. Os efeitos da temperatura de secagem nos coeficientes e nas constantes foram preditos pelos modelos de regressão. $\mathrm{O}$ modelo de Page modificado foi selecionado para representar o comportamento da secagem em camada delgada de bagaço de uva. Os valores médios da difusividade efetiva variaram de $1,0091 \times 10^{-9} \mathrm{~m}^{2} \mathrm{~s}^{-1}$ a $3,0421 \times 10^{-9} \mathrm{~m}^{2} \mathrm{~s}^{-1}$ nas temperaturas avaliadas. A dependência da difusividade efetiva pela temperatura foi descrita pela equação de Arrhenius, com o valor de energia de ativação de $24,512 \mathrm{~kJ} \mathrm{~mol}^{-1}$.
\end{abstract}

Termos para indexação: Vitis labrusca, difusividade efetiva, energia de ativação, produção de vinho, resíduo agroindustrial, taxa de secagem.

\section{Mathematical modeling of thin-layer drying of fermented grape pomace}

Abstract - The objective of this work was to determine the drying characteristics of fermented grape pomace in a hot air-dryer, to evaluate the descriptive capacity of known mathematical models of thin-layer drying, and to establish the values of effective diffusivity and activation energy. Drying experiments were conducted at 50, 60, 70,80 and $90^{\circ} \mathrm{C}$, with $1.0 \mathrm{~m} \mathrm{~s}^{-1}$ air-drying velocity. Ten different mathematical models for thin-layer drying were compared, according to the values of coefficient of determination $\left(\mathrm{R}^{2}\right)$, chi-square $\left(\chi^{2}\right)$, residual mean square root (RMSR), and relative mean error $(\mathrm{P})$ estimated by the drying curves. The effects of drying temperature on the coefficients and constants were predicted by the regression models. The modified Page model was selected to represent the behavior of thin-layer drying of grape pomace. The mean values of effective diffusivity varied from $1.0091 \times 10^{-9} \mathrm{~m}^{2} \mathrm{~s}^{-1}$ to $3.0421 \times 10^{-9} \mathrm{~m}^{2} \mathrm{~s}^{-1}$, at the evaluated temperatures. Effective diffusivity dependence on temperature was described by the Arrhenius equation, with activation energy of $24.512 \mathrm{~kJ} \mathrm{~mol}^{-1}$.

Index terms: Vitis labrusca, effective diffusivity, activation energy, wine production, agro-industrial residue, drying rate.

\section{Introdução}

As agroindústrias de alimentos produzem anualmente grande quantidade de resíduos líquidos e sólidos. Estes resíduos são constituídos, principalmente, por material orgânico biodegradável, e sua deposição gera sérios problemas ambientais. Apesar de poder ser aproveitada como ração animal ou disposta no campo, a maior parte dos resíduos agroindustriais ainda é descartada sem tratamento, com alto potencial de impacto ao meio ambiente (Hang, 2004; Makris et al., 2007b; Melo et al., 2011).
A produção de uvas no Brasil, em 2011, foi de 1.463.481 toneladas; deste total, $52,13 \%$ destinaramse à fabricação de vinhos, sucos e derivados (Mello, 2012). Na elaboração do vinho, o bagaço de uva é o principal subproduto e representa cerca de $20 \%$ do peso original das uvas (Gómez-Plaza et al., 2006; Llobera \& Cañellas, 2007; Ruberto et al., 2007). O bagaço de uva é formado, em média, por $58 \%$ de cascas, $20 \%$ de engaços e $22 \%$ de sementes (Dantas et al., 2008). O resíduo gerado pelas vinícolas tem grande quantidade de compostos fenólicos, bem conhecidos 
como antioxidantes naturais. Esses compostos são eficazes como anti-inflamatórios, anticancerígenos, antimutagênicos, antiúlcera, antiaterogênicos, agentes antimicrobianos e inibidores de oxidação de lipoproteínas de baixa densidade (Yi et al., 2009). Esse resíduo pode, portanto, ser aproveitado pela extração destas substâncias naturais, e ser utilizado nas indústrias de alimentos, cosméticos, química e farmacêutica, na forma de extratos antioxidantes naturais (Makris et al., 2007b; Gárcia-Perez et al., 2010). O bagaço de uva também é fonte significativa de fibras dietéticas, e alguns autores têm investigado a sua utilização na forma de farinha ou pó, como ingrediente na elaboração de biscoitos, snacks e produtos cárneos derivados de pescado e frango (Canett Romero et al., 2004; Sánchez-Alonso et al., 2007; Altan et al., 2008; Sáyago-Ayerdi et al., 2009).

O bagaço de uva possui teor de água que pode variar de 55 a 72\% em base úmida (Makris et al., 2007a), o que o torna altamente perecível. A secagem desse resíduo, em condições adequadas, por agroindústrias vinícolas, pode aumentar a vida útil e o aproveitamento do resíduo, reduzir o impacto ambiental e gerar benefícios econômicos (Gárcia-Perez et al., 2010).

Os métodos de secagem por convecção com ar aquecido e por liofilização são normalmente utilizados para alimentos perecíveis, assim como para o bagaço de uva (Katsube et al., 2009; Torres et al., 2010). A secagem por ar aquecido geralmente é preferida, em razão do custo e velocidade do processo (Katsube et al., 2009). Várias investigações foram feitas quanto às características de secagem de frutas, como figo (Babalis et al., 2006), maçã (Sacilik \& Elicin, 2006) e morango (Doymaz, 2008), e de resíduos agroindustriais como bagaço de maçã (Wang et al., 2007) e de semente de uva (Roberts et al., 2008; Celma et al., 2009).

Larrauri et al. (1997) estudaram o efeito da temperatura de secagem no teor de polifenóis e na atividade antioxidante de cascas de bagaço de uva fermentado, e observaram que não há perdas significativas nas características avaliadas, a $60^{\circ} \mathrm{C}$, em comparação à amostra de referência liofilizada. Roberts et al. (2008) estudaram a cinética de secagem por ar aquecido das sementes de uva, para posterior extração do óleo. No entanto, não foi encontrado nenhum trabalho sobre a cinética de secagem de bagaço de uva em sua totalidade, composto de cascas e sementes, forma normalmente obtida nas vinícolas.
A modelagem matemática das curvas de secagem e os valores da difusividade efetiva e energia de ativação são fundamentais para o projeto e construção de equipamentos de secagem (Celma et al., 2009).

O objetivo do presente trabalho foi determinar as características da secagem de bagaço de uva fermentado em secador com ar aquecido, avaliar a capacidade descritiva de modelos matemáticos de secagem em camada delgada, disponíveis na literatura, e obter os valores de difusividade efetiva e a energia de ativação.

\section{Material e Métodos}

Foram utilizadas uvas da variedade Isabel (Vitis labrusca), produzidas no Município de Santa Teresa, ES, a $19^{\circ} 56^{\prime} 08^{\prime \prime S}$ e $40^{\circ} 36^{\prime} 01^{\prime \prime} \mathrm{W}$, na safra $2007 / 2008$. As uvas foram recebidas no mesmo dia da colheita pela Cantina Matiello, também em Santa Teresa, e utilizadas imediatamente na elaboração de vinho tinto de mesa. Após a pesagem, higienização e seleção, as uvas passaram pelas etapas de desengace, esmagamento e fermentação. Após sete dias de fermentação, o mosto foi separado das cascas, sementes e algumas ráquis, com a utilização de prensa manual descontínua. O resíduo retido na prensa, denominado bagaço de uva, foi acondicionado em embalagens de polietileno e armazenado a $-18^{\circ} \mathrm{C}$, no mesmo dia, em freezer horizontal doméstico. O material congelado foi acondicionado em caixas de isopor e transportado para o Departamento de Tecnologia de Alimentos (DTA), da Universidade Federal de Viçosa (UFV), onde foi novamente armazenado a $-18^{\circ} \mathrm{C}$.

Os experimentos de secagem foram realizados no Laboratório de Frutas e Hortaliças, do DTA/ UFV. Utilizou-se um secador de camada delgada experimental, com ar aquecido. O secador é constituído por um ventilador centrífugo, um aquecedor elétrico, e uma câmara de secagem com estrutura de sustentação para três bandejas de alumínio, além de controlador de temperatura MT-511R, (Fullgauge, Canoas, RS). A velocidade do ar de secagem foi ajustada, por meio da manipulação da válvula de admissão de ar no secador, e medida com auxílio de um termoanemômetro digital portátil de fio quente modelo TAFR-180, (Instrutherm, São Paulo, SP).

Para determinação da curva de secagem, o bagaço congelado foi descongelado em refrigerador, com temperatura média de $8^{\circ} \mathrm{C}$, por 24 horas. Após o 
descongelamento, cerca de $100 \mathrm{~g}$ de bagaço de uva fermentado, à proporção de 70:30 para casca e semente, foram dispostos de forma homogênea em uma bandeja de alumínio, com fundo telado em malha fina. A espessura do bagaço foi de cerca de $10 \mathrm{~mm}$ (Wang et al., 2007). O secador foi colocado em funcionamento sem carga, durante $30 \mathrm{~min}$, para uniformização da temperatura do ar de secagem.

Foram realizados ensaios nas temperaturas de secagem $50,60,70,80$ e $90^{\circ} \mathrm{C}$, com velocidade de ar de $1,0 \mathrm{~m} \mathrm{~s}^{-1}$ e três repetições para cada temperatura, no total de 15 ensaios. O peso da bandeja com o bagaço de uva foi tomado a intervalos de $5 \mathrm{~min}$, durante a primeira hora de secagem, 10 min durante a segunda hora, $20 \mathrm{~min}$ durante a terceira hora, e a cada 30 min a partir de então, até que não houvesse variação significativa na massa da amostra (massa constante). Considerou-se, para efeito de cálculos, que a umidade de equilíbrio foi atingida quando não houve mais variação de peso. As pesagens ocorreram em balança eletrônica de precisão, da marca Marte, modelo AS 500, com precisão de 0,01 g. Ao final da secagem, uma parte da amostra foi retirada para determinação da massa de matéria seca. A matéria seca, a umidade inicial e a umidade de equilíbrio de cada um dos 15 ensaios foram determinadas em estufa a $105^{\circ} \mathrm{C}$, conforme Cunniff (1995).

Aos dados experimentais de secagem do bagaço de uva fermentado a diferentes temperaturas, foram ajustados dez modelos de secagem em camada delgada (Tabela 1). A razão de umidade (RU) foi calculada como $\mathrm{RU}=\left(\mathrm{U}-\mathrm{U}_{\mathrm{e}}\right) /\left(\mathrm{U}_{\mathrm{o}}-\mathrm{U}_{\mathrm{e}}\right)$, em que: $\mathrm{U}$ é a umidade em determinado tempo (em base seca); $U_{e}$ é a umidade

Tabela 1. Modelos matemáticos utilizados para descrever o processo de secagem.

\begin{tabular}{|c|c|}
\hline Nome do modelo & Equação $^{(1)}$ \\
\hline Newton & $R U=\exp (-k t)$ \\
\hline Page & $R U=\exp \left(-k t^{n}\right)$ \\
\hline Page modificado & $\mathrm{RU}=\exp \left[-(\mathrm{kt})^{\mathrm{n}}\right]$ \\
\hline Henderson e Pabis & $\mathrm{RU}=\mathrm{aexp}(-\mathrm{kt})$ \\
\hline Logarítmico & $\mathrm{RU}=\mathrm{a} \exp (-\mathrm{kt})+\mathrm{c}$ \\
\hline Dois termos & $R U=\operatorname{aexp}\left(-k_{0} t\right)+b \exp \left(-k_{1} t\right)$ \\
\hline Aproximação da difusão & $R U=a \exp (-k t)+(1-a) \exp (-k b t)$ \\
\hline Verma et al. (1985) & $R U=a \exp (-k t)+(1-a) \exp \left(-k_{1} t\right)$ \\
\hline Henderson e Pabis modificado & $R U=a \exp (-k t)+b \exp \left(-k_{0} t\right)+\operatorname{cexp}\left(-k_{1} t\right)$ \\
\hline Dois termos (exponencial) & $R U=a \exp (-k t)+(1-a) \exp (-k a t)$ \\
\hline
\end{tabular}

(1)RU é a razão de umidade; $k, k_{0}, k_{1}$ são as constantes de secagem por minuto; a, b, c, n são os coeficientes dos modelos; e té o tempo de secagem, em minutos. Fonte: Akpinar et al. (2003). de equilíbrio (em base seca); e $\mathrm{U}_{\mathrm{o}}$ é a umidade inicial (em base seca). A taxa de secagem do bagaço de uva fermentado foi dada por taxa de secagem $=\left(\mathrm{U}_{\mathrm{t}+\mathrm{dt}}-\mathrm{U}_{\mathrm{t}}\right) /$ (dt), em que: $U_{t}$ é a umidade no tempo $t$ (em base seca); $\mathrm{U}_{\mathrm{t}+\mathrm{dt}}$ é a umidade no tempo $\mathrm{t}+\mathrm{dt}$ (em base seca); dt é o acréscimo infinitesimal do tempo; e t é o tempo.

Os ajustes dos modelos matemáticos testados (Tabela 1) foram avaliados por meio da comparação dos valores de coeficiente de determinação $\left(\mathrm{R}^{2}\right)$, qui-quadrado $\left(\chi^{2}\right)$, raiz do quadrado médio residual (RQMR) e erro médio relativo (P). Os maiores valores de $\mathrm{R}^{2}$ e os menores valores de $\chi^{2}$ e RQMR indicam os melhores ajustes dos modelos. Enquanto os valores de RQMR e $\chi^{2}$ foram usados para comparar diferenças entre a razão de umidade experimental e a razão de umidade predita, os do erro médio relativo foram usados para comparar as diferenças absolutas entre o teor de água predito e o teor de água experimental durante a secagem (Roberts et al., 2008). Os valores de $\chi^{2}$ e RQMR são calculados por:

$$
\begin{aligned}
& \chi^{2}=\left(\sum_{\mathrm{i}=1}^{\mathrm{n}}\left(\mathrm{RU}_{\mathrm{exp}, \mathrm{i}}-\mathrm{RU_{ \textrm {pre } , \mathrm { i } }}\right)^{2}\right) /(\mathrm{N}-\mathrm{n}), \\
& \operatorname{RQMR}=\left[(1 / \mathrm{N}) \sum_{\mathrm{i}=1}^{\mathrm{N}}\left(\mathrm{RU}_{\text {pre }, \mathrm{i}}-\mathrm{RU}_{\text {exp,i }}\right)^{2}\right]^{1 / 2},
\end{aligned}
$$

em que: $\mathrm{RU}_{\text {exp,i }}$ é a i-ésima razão de umidade experimental; $\mathrm{RU}_{\text {pre,i, }}$ a i-ésima razão de umidade predita pelo modelo ajustado; N, o número de observações; e n, o número de parâmetros do modelo.

$\mathrm{O}$ erro médio relativo $(\mathrm{P})$ foi calculado como:

$$
\mathrm{P}=(100 / \mathrm{N}) \sum_{\mathrm{i}=1}^{\mathrm{N}}\left(\mathrm{U}_{\text {exp }, \mathrm{i}}-\mathrm{U}_{\mathrm{pre}, \mathrm{i}} / \mathrm{U}_{\mathrm{exp}, \mathrm{i}}\right)
$$

em que: $U_{\text {exp,i }}$ é o i-ésimo teor de água experimental em base seca; $\mathrm{U}_{\text {pre,i, o }} \mathrm{i}$-ésimo teor de água predito pelo modelo, ajustado em base seca; e $\mathrm{N}$, o número de observações. Um erro médio relativo abaixo de 10\% indica um bom ajuste do modelo (Mohapatra \& Rao, 2005).

A segunda lei de Fick foi utilizada para descrever o comportamento da secagem. A solução para a segunda lei de Fick, para placas planas - assumindo-se que a migração da umidade ocorre somente por difusão, que o encolhimento é insignificante, e que os coeficientes de 
difusão e a temperatura são constantes - é representada por:

$R U=\frac{U-U_{e}}{U_{0}-U_{e}}=\frac{8}{\pi^{2}} \sum_{i=1}^{\infty} \frac{1}{(2 i+1)^{2}} \exp \left(\frac{-(2 i+1)^{2} \pi^{2} D_{e f} t}{4 L^{2}}\right)$,

em que: RU é a razão de umidade; $\mathrm{U}$, a umidade em determinado tempo; $U_{e}$, a umidade de equilíbrio (base seca); $U_{o}$, a umidade inicial (base seca); Def, a difusividade efetiva $\left(\mathrm{m}^{2} \mathrm{~s}^{-1}\right)$; $\mathrm{L}$, a semiespessura da camada do bagaço de uva (m); e t, o tempo (s).

Para longos períodos de secagem, essa equação pode ser simplificada para somente o primeiro termo da série, conforme:

$\ln (\mathrm{RU})=\ln \left(\frac{8}{\pi^{2}}-\frac{\pi^{2} \mathrm{D}_{\mathrm{ef}}}{4 \mathrm{~L}^{2}} \times \mathrm{t}\right)$

em que: $\mathrm{RU}$ é a razão de umidade; $\mathrm{D}_{\text {ef, }}$ a difusividade efetiva $\left(\mathrm{m}^{2} \mathrm{~s}^{-1}\right)$; L, a semiespessura da camada do bagaço de uva (m); e t, o tempo (s).

Os valores de difusividade efetiva foram determinados pela plotagem dos dados de secagem experimental, em termos do logaritmo natural da razão de umidade pelo tempo de secagem da equação simplificada. O gráfico resultante fornece a seguinte reta de inclinação:

Inclinação $=\frac{\pi^{2} \mathrm{D}_{\mathrm{ef}}}{4 \mathrm{~L}^{2}}$.

A dependência da difusividade efetiva pela temperatura foi expressa por uma relação do tipo equação de Arrhenius, como segue:

$\mathrm{D}_{\mathrm{ef}}=\mathrm{D}_{0} \exp \left(-\frac{\mathrm{E}_{\mathrm{a}}}{\mathrm{RT}}\right)$,

em que: $\mathrm{D}_{0}$ é o fator pré-exponencial da equação de Arrhenius $\left(\mathrm{m}^{2} \mathrm{~s}^{-1}\right) ; \mathrm{E}_{\mathrm{a}}$, a energia de ativação $\left(\mathrm{kJ} \mathrm{mol}^{-1}\right)$; $\mathrm{R}$, a constante universal dos gases $\left(\mathrm{kJ} \mathrm{mol}^{-1} \mathrm{~K}\right)$; e T, a temperatura absoluta $(\mathrm{K})$.

Da inclinação da reta do $\ln \left(\mathrm{D}_{\mathrm{ef}}\right)$ pelo recíproco da temperatura $(\mathrm{K})$, conforme descrito pela equação de Arrhenius, a energia de ativação foi calculada.

As variáveis razão de umidade e tempo de secagem foram ajustadas por meio da aplicação do método de mínimos quadrados, implementado no programa Statistica, versão 6 (StatSoft, 1998), para os modelos lineares e não lineares.

\section{Resultados e Discussão}

O conteúdo de umidade inicial do bagaço de uva, após o descongelamento, foi de cerca de 2,21 $\pm 0,03 \mathrm{~g}$ de água por $\mathrm{g}$ de matéria seca $\left(\mathrm{g} \mathrm{g}^{-1}\right)$. A umidade de equilíbrio foi de $0,063 \pm 0,001,0,057 \pm 0,001$, $0,047 \pm 0,001,0,045 \pm 0,002$ e $0,038 \pm 0,001 \mathrm{~g} \mathrm{~g}^{-1}$, à 50 , $60,70,80$ e $90^{\circ} \mathrm{C}$, respectivamente. O tempo necessário para atingir a umidade de equilíbrio, para o bagaço de uva fermentado, foi de 840, 600, 420, 330 e $270 \mathrm{~min}$, para $50,60,70,80$ e $90^{\circ} \mathrm{C}$, respectivamente (Figura 1). Como era esperado, o tempo de secagem foi menor quando a temperatura foi maior, o que é explicado pelo aumento da taxa de secagem (Akpinar et al., 2003). Este comportamento ocorre em virtude do aumento do potencial de transferência de calor entre o ar e a camada de bagaço de uva.

A redução do tempo de secagem, para cada intervalo de $10^{\circ} \mathrm{C}$ na faixa de temperatura estudada, foi de 28,6 , $30,0,21,4$ e 18,2\%. A maior redução no tempo de secagem $(30 \%)$ ocorreu no intervalo de temperatura entre 60 e $70^{\circ} \mathrm{C}$.

Pode-se notar que a taxa de secagem diminui continuamente com o tempo (Figura 2). Não foi observado nenhum período de secagem à taxa constante, o que indica que todo o processo ocorreu

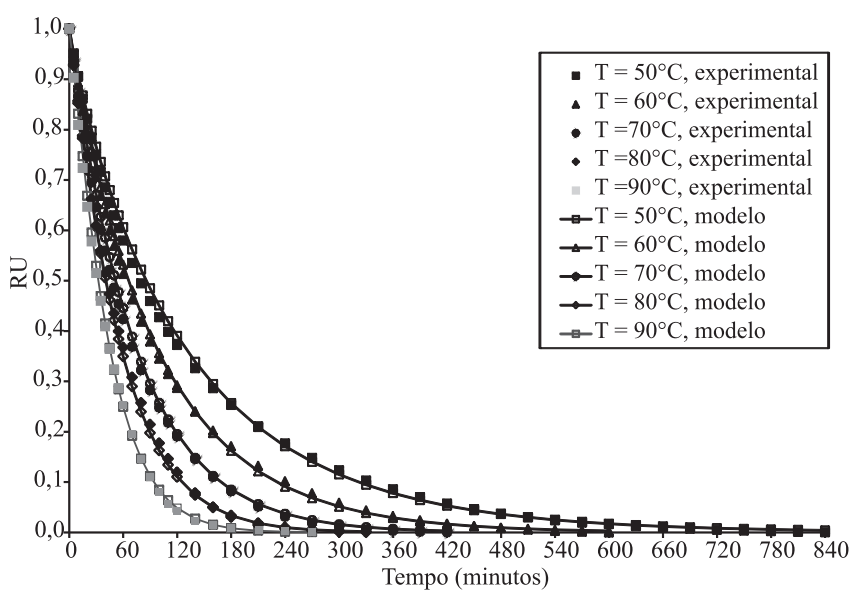

Figura 1. Curvas experimentais da secagem de camada delgada de bagaço de uva fermentado em função do tempo, preditas pelo modelo de Page modificado, a diferentes temperaturas. A razão de umidade (RU) foi calculada como $R U=\left(U-U_{e}\right) /\left(U_{o}-U_{e}\right)$, em que: $U$ é a umidade em determinado tempo (em base seca); $U_{e}$ é a umidade de equilíbrio (em base seca); e $\mathrm{U}_{\mathrm{o}}$ é a umidade inicial (em base seca). 
com taxa decrescente. Estes resultados estão de acordo com os de Panchariya et al. (2002), Baballis et al. (2006) e Doymaz (2008), na secagem de chá-preto, figo e morango, respectivamente. A taxa de secagem aumentou com o aumento de temperatura do ar de secagem, e o maior valor foi obtido no experimento a $90^{\circ} \mathrm{C}$, nos primeiros $60 \mathrm{~min}$ de secagem. Após $90 \mathrm{~min}$, a taxa de secagem a $90^{\circ} \mathrm{C}$ atingiu valor menor do que a temperaturas de $50,60,70$ e $80^{\circ} \mathrm{C}$. A taxa de secagem diminui continuamente com a diminuição do teor de água ou com o aumento do tempo de secagem (Sacilik \& Elicin, 2006).

Os dados do teor de água obtidos a diferentes temperaturas no experimento foram convertidos à razão de umidade e ajustados para os dez modelos (Tabela 1). Em todos os casos os valores de $\mathrm{R}^{2}$ foram superiores a 0,9214 (Tabela 2). Os maiores valores médios de $\mathrm{R}^{2}$ (acima de 0,9987 ) foram obtidos para os modelos de Page, Page modificado, aproximação da difusão e modelo de Verma et al. (1985), com valores médios de $\chi^{2}$ e RQMR abaixo de $2,53 \times 10^{-4}$ e 0,0149 , respectivamente (Tabela 2.). Esses quatro modelos também apresentaram menores valores nos percentuais de erro médio, com valores inferiores ou ligeiramente superiores a $10 \%$, o que indica, de acordo com Mohapatra \& Rao (2005), serem adequados à

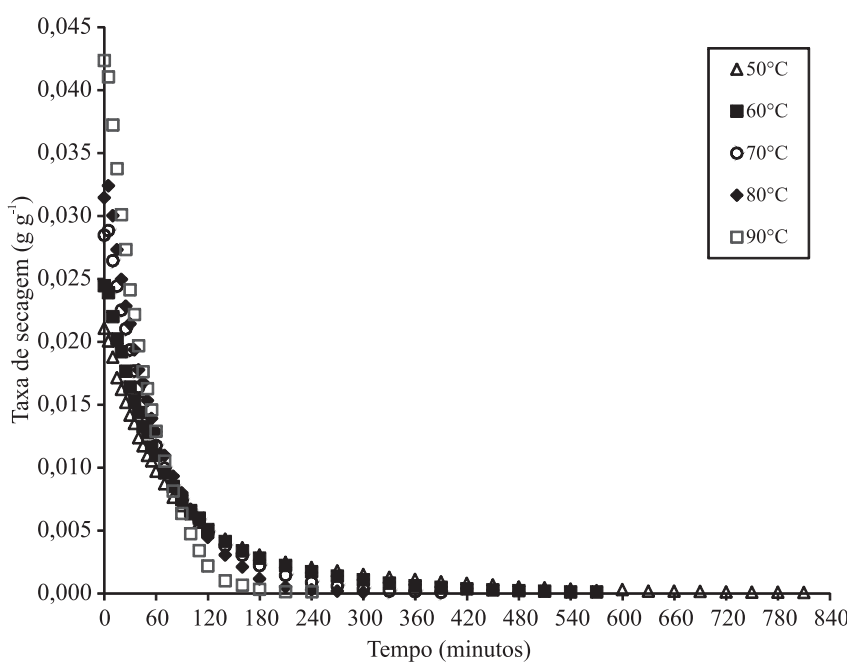

Figura 2. Taxa de secagem, em função do tempo de secagem do bagaço de uva fermentado a diferentes temperaturas. A taxa de secagem do bagaço de uva fermentado foi determinada pela equação taxa de secagem $=\left(\mathrm{U}_{\mathrm{t}+\mathrm{dt}}-\mathrm{U}_{\mathrm{t}}\right)$ / (dt), em que: $\mathrm{U}_{\mathrm{t}}$ é a umidade no tempo $\mathrm{t}$ (em base seca); $\mathrm{U}_{\mathrm{t}+\mathrm{dt}}$ é a umidade no tempo $\mathrm{t}+\mathrm{dt}$ (em base seca); e t é o tempo. descrição deste fenômeno. Os modelos de aproximação da difusão e o de Verma et al. (1985) são modelos de três coeficientes, enquanto os modelos de Page e Page modificado apresentam apenas dois coeficientes ( $\mathrm{k}$ e $\mathrm{n}$ ). Portanto, esses últimos são normalmente preferidos, por sua simplicidade. No entanto, estes coeficientes indicaram relação do teor de água com a faixa de temperatura estudada somente no modelo de Page modificado, e apresentaram valores crescentes com o aumento da temperatura (Tabela 2). Assim, o modelo de Page modificado foi selecionado para representar o comportamento da secagem, em camada delgada de bagaço de uva.

Com o intuito de avaliar o efeito da temperatura de secagem nos coeficientes $\mathrm{k}$ e $\mathrm{n}$ do modelo de Page modificado, análises de regressão foram realizadas para avaliar a relação entre estes parâmetros e a temperatura. As equações de regressão destes parâmetros com a temperatura e os modelos obtidos seguiram o modelo:

$\mathrm{RU}=\exp \left[-(\mathrm{kt})^{\mathrm{n}}\right]$,

em que RU é a razão de umidade e t o tempo, com os seguintes valores: $\mathrm{k}=-0,0000078+0,00014 \mathrm{~T}$ $-0,00000078 \mathrm{~T}^{2}+0,000000022 \mathrm{~T}^{3} \quad\left(\mathrm{R}^{2}=0,9987\right) \mathrm{e}$ $\mathrm{n}=(0,0000056+0,14 \mathrm{~T}) /\left(1+0,16 \mathrm{~T}-0,00052 \mathrm{~T}^{2}\right), \mathrm{R}^{2}=0,999$.

Assim, RU pôde ser descrita da seguinte forma:

$\mathrm{RU}=\exp \left[-\left(\left(-7,8 \times 10^{-6}+1,4 \times 10^{-4} \mathrm{~T}-7,8 \times 10^{-6} \mathrm{~T}^{2}\right.\right.\right.$

$\left.\left.\left.+2,2 \times 10^{-8} \mathrm{~T}^{3}\right) \times \mathrm{t}\right)^{\left(5,6 \times 10^{-6}+0,14 \mathrm{~T}\right) /\left(1+0,16 \mathrm{~T}-5,2 \times 10^{-4} \mathrm{~T}^{2}\right)}\right]$

em que: T é a temperatura absoluta (K); e t, o tempo. A consistência do modelo e as relações entre os coeficientes e a temperatura de secagem foram evidentes com $\mathrm{R}^{2}=0,9983 ; \chi^{2}=0,00037$ e $\mathrm{RQMR}=0,0192$.

A precisão do modelo estabelecido foi avaliada por meio da comparação da razão de umidade, calculada pelo modelo de Page modificado, com a razão de umidade observada em todas as temperaturas avaliadas, em que foi verificada uma excelente concordância entre os dados experimentais e os calculados pelo modelo (Figura 1). Assim, a razão de umidade do bagaço de uva fermentado, em qualquer tempo, durante o processo de secagem em camada delgada, pode ser estimada com maior precisão com a utilização desta expressão.

Os resultados experimentais mostraram que a resistência à transferência de massa interna controla o tempo de secagem, em virtude da presença de um 
Tabela 2. Resultados obtidos nos diferentes modelos de secagem testados.

\begin{tabular}{|c|c|c|c|c|c|c|}
\hline Modelo & Temperatura $\left({ }^{\circ} \mathrm{C}\right)$ & Constantes $^{(1)}$ & $\mathrm{R}^{2}$ & $\chi^{2}$ & RQMR & $\mathrm{P}$ \\
\hline \multirow{5}{*}{ Newton } & 50 & $\mathrm{k}=0,00787$ & 0,9959 & $8,78 \times 10^{-4}$ & 0,0295 & 10,76 \\
\hline & 60 & $\mathrm{k}=0,01072$ & 0,9973 & $5,56 \times 10^{-4}$ & 0,0235 & 7,81 \\
\hline & 70 & $\mathrm{k}=0,01423$ & 0,9996 & $7,35 \times 10^{-5}$ & 0,0085 & 4,71 \\
\hline & 80 & $k=0,01692$ & 0,9987 & $2,47 \times 10^{-4}$ & 0,0156 & 11,76 \\
\hline & 90 & $\mathrm{k}=0,02285$ & 0,9986 & $2,63 \times 10^{-4}$ & 0,0161 & 13,32 \\
\hline \multirow{5}{*}{ Page } & 50 & $\mathrm{k}=0,01348 \quad \mathrm{n}=0,88521$ & 0,9977 & $4,98 \times 10^{-4}$ & 0,0222 & 10,08 \\
\hline & 60 & $\mathrm{k}=0,01546 \quad \mathrm{n}=0,91666$ & 0,9982 & $3,73 \times 10^{-4}$ & 0,0191 & 8,03 \\
\hline & 70 & $\mathrm{k}=0,01561 \quad \mathrm{n}=0,97785$ & 0,9997 & $6,17 \times 10^{-5}$ & 0,0078 & 5,73 \\
\hline & 80 & $\mathrm{k}=0,01393 \quad \mathrm{n}=1,04822$ & 0,9990 & $1,94 \times 10^{-4}$ & 0,0137 & 7,94 \\
\hline & 90 & $\mathrm{k}=0,01700 \quad \mathrm{n}=1,07738$ & 0,9993 & $1,37 \times 10^{-4}$ & 0,0116 & 6,18 \\
\hline \multirow{5}{*}{ Page modificado } & 50 & $\mathrm{k}=0,00771 \quad \mathrm{n}=0,88525$ & 0,9977 & $4,98 \times 10^{-4}$ & 0,0222 & 10,08 \\
\hline & 60 & $\mathrm{k}=0,01058 \quad \mathrm{n}=0,91668$ & 0,9982 & $3,73 \times 10^{-4}$ & 0,0191 & 8,03 \\
\hline & 70 & $\mathrm{k}=0,01421 \quad \mathrm{n}=0,97790$ & 0,9997 & $6,17 \times 10^{-5}$ & 0,0078 & 5,73 \\
\hline & 80 & $\mathrm{k}=0,01695 \quad \mathrm{n}=1,04828$ & 0,9990 & $1,94 \times 10^{-4}$ & 0,0137 & 7,94 \\
\hline & 90 & $\mathrm{k}=0,02278 \quad \mathrm{n}=1,07743$ & 0,9993 & $1,37 \times 10^{-4}$ & 0,0116 & 6,17 \\
\hline \multirow{5}{*}{ Henderson e Pabis } & 50 & $\mathrm{k}=0,00738 \quad \mathrm{n}=0,96187$ & 0,9967 & $7,08 \times 10^{-4}$ & 0,0264 & 8,99 \\
\hline & 60 & $\mathrm{k}=0,01031 \quad \mathrm{n}=0,97469$ & 0,9976 & $4,85 \times 10^{-4}$ & 0,0218 & 6,80 \\
\hline & 70 & $\mathrm{k}=0,01412 \quad \mathrm{n}=0,99418$ & 0,9996 & $7,03 \times 10^{-5}$ & 0,0083 & 4,88 \\
\hline & 80 & $\mathrm{k}=0,01720 \quad \mathrm{n}=1,01291$ & 0,9988 & $2,30 \times 10^{-4}$ & 0,0150 & 10,92 \\
\hline & 90 & $\mathrm{k}=0,02336 \quad \mathrm{n}=1,01971$ & 0,9988 & $2,26 \times 10^{-4}$ & 0,0148 & 11,82 \\
\hline & 50 & $\mathrm{n}=0,01285 \quad \mathrm{a}=0,95502$ & 0,9969 & $6,67 \times 10^{-4}$ & 0,0255 & 13,83 \\
\hline & 60 & $\mathrm{n}=0,01051 \quad \mathrm{a}=0,96863$ & 0,9978 & $4,61 \times 10^{-4}$ & 0,0212 & 11,11 \\
\hline Logarítmico & 70 & $\mathrm{n}=-0,00050 \quad \mathrm{a}=0,99448$ & 0,9996 & $7,11 \times 10^{-5}$ & 0,0083 & 4,57 \\
\hline & 80 & $\mathrm{n}=-0,01371 \quad \mathrm{a}=1,02176$ & 0,9990 & $1,97 \times 10^{-4}$ & 0,0138 & 7,95 \\
\hline & 90 & $\mathrm{n}=-0,01536 \quad \mathrm{a}=1,02939$ & 0,9991 & $1,76 \times 10^{-4}$ & 0,0130 & 13,43 \\
\hline & 50 & $a=-0,00403 \quad b=0,00000$ & 0,9230 & $4,86 \times 10^{-4}$ & 0,1256 & 9,81 \\
\hline & 60 & $a=-0,00582 \quad b=0,00001$ & 0,9215 & $3,66 \times 10^{-4}$ & 0,1233 & 8,37 \\
\hline Dois termos & 70 & $a=-0,00834$ & 0,9402 & $5,99 \times 10^{-5}$ & 0,1059 & 5,64 \\
\hline & 80 & $\mathrm{a}=-0,01040$ & 0,9574 & $2,36 \times 10^{-4}$ & 0,0898 & 11,01 \\
\hline & 90 & $a=-0,01319 \quad b=0,00004$ & 0,9422 & $2,33 \times 10^{-4}$ & 0,1023 & 11,78 \\
\hline & 50 & $\mathrm{k}=0,02228$ & 0,9978 & $4,82 \times 10^{-4}$ & 0,0217 & 9,85 \\
\hline & 60 & $\mathrm{k}=0,02520$ & 0,9983 & $3,63 \times 10^{-4}$ & 0,0188 & 8,40 \\
\hline Aproximação da difusão & 70 & $\mathrm{a}=0,04585 \quad \mathrm{~b}=0,32265$ & 0,9997 & $6,01 \times 10^{-5}$ & 0,0076 & 5,62 \\
\hline & 80 & $a=-16,29861 \quad b=0,98177$ & 0,9991 & $1,87 \times 10^{-4}$ & 0,0134 & 6,90 \\
\hline & 90 & $a=-78,87201 \quad b=0,99516$ & 0,9994 & $1,25 \times 10^{-4}$ & 0,0110 & 5,01 \\
\hline & 50 & $\mathrm{a}=0,21973 \quad \mathrm{k}_{1}=0,00609$ & 0,9978 & $4,82 \times 10^{-4}$ & 0,0217 & 9,85 \\
\hline & 60 & $\mathrm{a}=0,78308 \quad \mathrm{k}_{1}=0,02520$ & 0,9983 & $3,63 \times 10^{-4}$ & 0,0188 & 8,40 \\
\hline Verma et al. (1985) & 70 & $\mathrm{a}=0,95398 \quad \mathrm{k}_{1}=0,04205$ & 0,9997 & $6,01 \times 10^{-5}$ & 0,0076 & 5,62 \\
\hline & 80 & $\mathrm{a}=-1,89805 \mathrm{k}_{1}=0,01322$ & 0,9991 & $1,81 \times 10^{-4}$ & 0,0132 & 6,32 \\
\hline & 90 & $k=0,01519 \quad a=-2,68523 \quad k_{1}=0,01696$ & 0,9993 & $1,36 \times 10^{-4}$ & 0,0114 & 10,73 \\
\hline & 50 & $\begin{array}{ll}\mathrm{a}=0,21785 & \mathrm{~b}=0,39434 \\
\mathrm{k}_{0}=0,00610 & \mathrm{k}_{1}=0,00610\end{array}$ & 0,9978 & $4,93 \times 10^{-4}$ & 0,0217 & 9,82 \\
\hline & 60 & $\begin{array}{lll}\mathrm{k}=0,00861 & \mathrm{a}=0,04390 & \mathrm{~b}=0,74756 \\
\mathrm{c}=0,21118 & \mathrm{k}_{0}=0,00870 & \mathrm{k}_{1}=0,02630\end{array}$ & 0,9983 & $3,73 \times 10^{-4}$ & 0,0188 & 8,37 \\
\hline Henderson e Pabis modificado & 70 & $\begin{array}{lll}\mathrm{k}=0,01362 & \mathrm{a}=0,86609 & \mathrm{~b}=0,08900 \\
\mathrm{c}=0,04876 & \mathrm{k}_{0}=0,01359 & \mathrm{k}_{1}=0,04710\end{array}$ & 0,9997 & $6,14 \times 10^{-5}$ & 0,0076 & 5,64 \\
\hline & 80 & $\begin{array}{ll}\mathrm{a}=0,33755 & \mathrm{~b}=0,33755 \\
\mathrm{k}_{0}=0,01719 & \mathrm{k}_{1}=0,01719\end{array}$ & 0,9988 & $2,43 \times 10^{-4}$ & 0,0150 & 10,95 \\
\hline & 90 & $\begin{array}{lll}\mathrm{k}=0,02342 & \mathrm{a}=0,34248 & \mathrm{~b}=0,33850 \\
\mathrm{c}=0,33850 & \mathrm{k}_{0}=0,02333 & \mathrm{k}_{1}=0,02332\end{array}$ & 0,9988 & $2,40 \times 10^{-4}$ & 0,0148 & 11,84 \\
\hline & 50 & $k=0,01777 \quad a=0,31525$ & 0,9977 & $4,88 \times 10^{-4}$ & 0,0219 & 11,99 \\
\hline & 60 & $\mathrm{k}=0,20883 \quad \mathrm{a}=0,04770$ & 0,9978 & $4,44 \times 10^{-4}$ & 0,0209 & 6,36 \\
\hline Dois termos (exponencial) & 70 & $\mathrm{k}=1,47420 \quad \mathrm{a}=0,00953$ & 0,9997 & $6,89 \times 10^{-5}$ & 0,0082 & 4,99 \\
\hline & 80 & $\mathrm{k}=4,32885 \quad \mathrm{a}=0,00389$ & 0,9987 & $2,63 \times 10^{-4}$ & 0,0160 & 12,10 \\
\hline
\end{tabular}

${ }^{(1)}$ Determinação em triplicata para cada temperatura; $\mathrm{R}^{2}$, coeficiente de determinação; RQMR, raiz do quadrado médio residual; $\chi^{2}$, valor de qui-quadrado; $\mathrm{P}$, erro médio relativo. 
período de secagem à taxa decrescente (Wang et al., 2007). Portanto, os valores da difusividade efetiva, a diferentes temperaturas, podem ser obtidos a partir da segunda lei de Fick, com sua equação simplificada, e pela equação que define a inclinação dessa função. Os valores da difusividade efetiva, em $\mathrm{m}^{2} \mathrm{~s}^{-1}$, foram de $1,0091 \times 10^{-9}, 1,3250 \times 10^{-9}, 1,8148 \times 10^{-9}, 2,1987$ x $10^{-9}$ e $3,0421 \times 10^{-9}$, para as temperaturas de 50,60, 70,80 e $90^{\circ} \mathrm{C}$, respectivamente. Estes valores indicam a velocidade com que a água sai do produto em estudo. Como era esperado, os valores de difusividade efetiva aumentaram com o aumento da temperatura.

Estes resultados estão de acordo com os obtidos por Wang et al. (2007) para a secagem de bagaço de maçã, em que obtiveram valores de difusividade efetiva na faixa de $1,9082 \times 10^{-9}$ a $3,9346 \times 10^{-9} \mathrm{~m}^{2} \mathrm{~s}^{-1}$ para temperaturas de 75 a $105^{\circ} \mathrm{C}$. Roberts et al. (2008) também observaram este efeito para a secagem de semente de bagaço de uva de três variedades diferentes, tendo obtido valores de Def $\left(\mathrm{m}^{2} \mathrm{~s}^{-1}\right)$ menores - de $1,57 \times 10^{-10}$ a $8,03 \times 10^{-10}$ - para temperaturas de 40 a $60^{\circ} \mathrm{C}$. Essas menores difusividades efetivas podem ser explicadas pela composição do bagaço de uva. No presente trabalho, este foi constituído de 30\% de sementes e o restante de cascas.

Observou-se relação linear, derivada da equação de Arrhenius, do logaritmo natural de Def em função da temperatura absoluta (Figura 3). Da inclinação da reta, obteve-se o valor de energia de ativação de $26,440 \mathrm{~kJ} \mathrm{~mol}^{-1}$. Wang et al. (2007) obtiveram um valor próximo, $24,512 \mathrm{~kJ} \mathrm{~mol}^{-1}$, para a energia de ativação do bagaço de maçã. Roberts et al. (2008) obtiveram valores de energia de ativação na faixa de 30 a $40 \mathrm{~kJ}$ $\mathrm{mol}^{-1}$, na secagem de semente de bagaço de uva de três variedades diferentes. Os maiores valores de energia

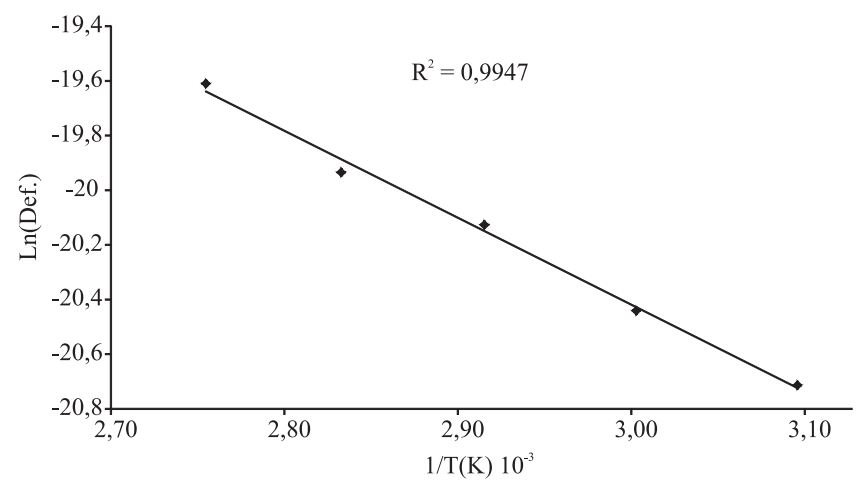

Figura 3. Efeito da temperatura $[1 / \mathrm{T}(\mathrm{K})]$ na difusividade efetiva $[\ln ($ Def.) $]$ de bagaço de uva fermentado. de ativação para a semente, e consequente menor difusividade efetiva, obtidos por esses autores, podem ser justificados pelo fato de a estrutura da semente apresentar maior resistência interna à transferência de massa do que a casca.

\section{Conclusões}

1. A secagem em camada delgada de bagaço de uva é mais bem descrita pelo modelo de Page modificado.

2. Todo o processo de secagem do bagaço de uva ocorre à taxa decrescente; e não há, portanto, período de taxa constante de secagem.

3. Os valores médios de difusividade efetiva variam de $1,0091 \times 10^{-9} \mathrm{~m}^{2} \mathrm{~s}^{-1}$ a $3,0421 \times 10^{-9} \mathrm{~m}^{2} \mathrm{~s}^{-1}$ para a secagem de bagaço de uva entre 50 e $90^{\circ} \mathrm{C}$, respectivamente.

4. A dependência da difusividade efetiva pela temperatura é descrita pela equação de Arrhenius, com valor de energia de ativação de $24,512 \mathrm{~kJ} \mathrm{~mol}^{-1}$.

\section{Referências}

AKPINAR, E.K.; BICER, Y.; YILDIZ, C. Thin layer drying of red pepper. Journal of Food Engineering, v.59, p.99-104, 2003.

ALTAN, A.; MCCARTHY, K.L.; MASKAN, M. Twin-screw extrusion of barley-grape pomace blends: extrudate characteristics and determination of optimum processing conditions. Journal of Food Engineering, v.89, p.24-32, 2008.

CUNNIFF, P. Official methods of analysis of AOAC International. $16^{\text {th }}$ ed. Arlington: AOAC International, 1995.

BABALIS, S.J.; PAPANICOLAOU, E.; KYRIAKIS, N.; BELESSIOTIS, V.G. Evaluation of thin-layer drying models for describing drying kinetics of figs (Ficus carica). Journal of Food Engineering, v.75, p.205-214, 2006.

CANETT ROMERO, R.C.; OSUNA, A.I.L.; ROBLES, R.M.; CASTRO, S.R.M.; MARTÍNEZ, L.L.; LEÓN-GÁLVEZ, R. Caracterización de galletas elaboradas com cascarilla de orujo de uva. Archivos Latinoamericanos de Nutrición, v.54, p.93-199, 2004.

CELMA, A.R.; LÓPEZ-RODRIGUÉZ, F.; BLÁZQUEZ, C. Experimental modelling of infrared drying of industrial grape by-products. Food and Bioproducts Processing, v.87, p.247-253, 2009.

DANTAS, F.R.; ARAÚJO, G.G.L. de; SILVA, D.S. da; PEREIRA, L.G.R.; GONZAGA NETO, S.; TOSTO, M. da S.L. Composição química e características fermentativas de silagens de maniçoba (Manihot sp.) com porcentuais de co-produto de vitivinícolas desidratado. Revista Brasileira de Saúde e Produção Animal, v.9, p.247-257, 2008.

DOYMAZ, I. Convective drying kinetics of strawberry. Chemical Engineering and Processing, v.47, p.914-919, 2008. 
GÁRCIA-PEREZ, J.V.; GÁRCIA-ALVARADO, M.A.; CARCEL, J.A.; MULLET, A. Extraction kinetics modeling of antioxidants from grape stalk (Vitis vinifera var. Bobal): influence of drying conditions. Journal of Food Engineering, v.101, p.49-58, 2010.

GÓMEZ-PLAZA, E.; MIÑANO, A.; LÓPEZ-ROCA, J.M. Comparison of chromatic properties, stability and antioxidant capacity of anthocyanin-based aqueous extracts from grape pomace obtained from different vinification methods. Food Chemistry, v.97, p.87-94, 2006.

HANG, Y.D. Management and utilization of food processing wastes. Journal of Food Science, v.69, p.104-107, 2004.

KATSUBE, T.; TSURUNAGA, Y.; SUGIYAMA, M.; FURUNO, T.; YAMASAKI, Y. Effect of air-drying temperature on antioxidant capacity and stability of polyphenolic compounds in mulberry (Morus alba L.) leaves. Food Chemistry, v.113, p.964-969, 2009.

LARRAURI, J.A.; RUPÉREZ, P.; SAURA-CALIXTO, F. Effect of drying temperature on the stability of polyphenols and antioxidant activity of red grape pomace peels. Journal of Agricultural and Food Chemistry, v.45, p.1390-1393, 1997.

LLOBERA, A.; CAÑELLAS, J. Dietary fibre content and antioxidant activity of Manto Negro red grape (Vitis vinifera): pomace and stem. Food Chemistry, v.101, p.659-666, 2007.

MAKRIS, D.P.; BOSKOU, G.; ANDRIKOPOULOS, N.K. Polyphenolic content and in vitro antioxidant characteristics of wine industry and other agri-food solid waste extracts. Journal of Food Composition and Analysis, v.20, p.125-132, 2007a.

MAKRIS, D.P.; BOSKOU, G.; ANDRIKOPOULOS, N.K. Recovery of antioxidant phenolics from white vinification solid by-products employing water/ethanol mixtures. Bioresource Technology, v.98, p.2963-2967, 2007 b.

MELLO, L.M.R. Viticultura brasileira: panorama 2011. Bento Gonçalves: Embrapa Uva e Vinho, 2012. (Embrapa Uva e Vinho. Comunicado técnico, 115).

MELO, P.S.; BERGAMASCHI, K.B.; TIVERON, A.P.; MASSARIOLI, A.P.; OLDONI, T.L.C.; ZANUS, M.C.; PEREIRA, G.E.; ALENCAR, S.M. Composição fenólica e atividade antioxidante de resíduos agroindustriais. Ciência Rural, v.41, p.1088-1093, 2011.

MOHAPATRA, D.; RAO, P.S. A thin layer drying model of parboiled wheat. Journal of Food Engineering, v.66, p.513-518, 2005.
PANCHARIYA, P.C.; POPOVIC, D.; SHARMA, A.L. Thin-layer modelling of black tea drying process. Journal of Food Engineering, v.52, p.349-357, 2002.

ROBERTS, J.S.; KIDD, D.R.; PADILLA-ZAKOUR, O. Drying kinetics of grape seeds. Journal of Food Engineering, v.89, p.460-465, 2008.

RUBERTO, G.; RENDA, A.; DAQUINO, C.; AMICO, V.; SPATAFORA, C.; TRINGALI, C.; DE TOMMASI, N. Polyphenol constituents and antioxidant activity of grape pomace extracts from five sicilian red grape cultivars. Food Chemistry, v.100, p.203-210, 2007.

SACILIK, K.; ELICIN, A.K. The thin layer drying characteristics of organic apple slices. Journal of Food Engineering, v.73, p.281-289, 2006.

SÁNCHEZ-ALONSO, I.; JIMÉNEZ-ESCRIG, A.; SAURA-CALIXTO, F.; BORDERÍAS, A. J. Effect of grape antioxidant dietary fibre on the prevention of lipid oxidation in minced fish: evaluation by different methodologies. Food Chemistry, v.101, p.372-378, 2007.

SÁYAGO-AYERDI, S.G.; BRENES, A.; GOÑI, I. Effect of grape antioxidant dietary fiber on the lipid oxidation of raw and cooked chicken hamburgers. LWT - Food Science and Technology, v.42, p.971-976, 2009.

STATSOFT. Statistica for Windows. Version 6.0. Tulsa: StatSoft, 1998.

TORRES, C. de; DÍAZ-MAROTO, M.C.; HERMOSÍNGUTIÉRREZ, I.; PÉREZ-COELHO, M.S. Effect of freeze-drying and oven-drying on volatiles and phenolics composition of grape skin. Analytica Chimica Acta, v.660, p.177-182, 2010.

VERMA, L.R.; BUCKLIN, R.A.; ENDAN, J.B.; WRATTEN, F.T. Effects of drying air parameters on rice drying models. Transactions of the ASAE, v.28, p.296-301, 1985.

WANG, Z.; SUN, J.; LIAO, X.; CHEN, F.; ZHAO, G.; WU, J.; HU, X. Mathematical modeling on hot air drying of thin layer apple pomace. Food Research International, v.40, p.39-46, 2007.

YI, C.; SHI, J.; KRAMER, J.; XUE, S.; JIANG, Y.; ZHANG, M.; MA, Y.; POHORLY, J. Fatty acid composition and phenolic antioxidants of winemaking pomace powder. Food Chemistry, v.114, p.570-576, 2009.

Recebido em 12 de fevereiro de 2012 e aprovado em 25 de maio de 2012 